1999 Joint Meeting EFTF - IEEE IFCS

\title{
GROUP-DELAY ERRORS DUE TO COHERENT INTERFERENCE
}

\author{
F.G. ASCARRUNZ: . T.E. PARKER ${ }^{\dagger}$ and S.R. JEFFERTS ${ }^{\dagger}$ \\ "University of Colorado. Boulder CO. USA \\ ${ }^{\dagger}$ NIST Time and Frequency Division \\ 325 Broadway. Boulder CO. USA
}

\begin{abstract}
Time and frequency transfer techniques that rely on spread spectrum group-delay measurements are prone to potentially large timing errors caused by coherent interference that is time delayed. Reducing the level of the coherent interference. increasing the pseudo-random code rate, or using a fractional correlator can minimize these timing errors.
\end{abstract}

\section{INTRODUCTION}

The time-and-frequency community uses spread spectrum communication links for the transfer of time and frequency information from laboratory to laboratory or more commonly, the dissemination of time and frequency to users across the world. The two systems that are widely used for the transfer of timeand-frequency information are the global positioning system (GPS) and two-way satellite time-and-frequency transfer (TWSTFT). In both of these systems a timing signal is encoded on a carrier signal using bi-phase spread spectrum modulation. Data taken over the past ten years indicate that although TWSTFT is a two-way system where there should be common mode rejection of path delays, the delay stability of this method of transfer has generally not been much better than common view GPS. However, there is a recent TWSTFT link that shows a factor of 5 improvement in stability over other TWSTFT links [1]. In this paper we discuss limitations on time and frequency transfer due to fundamental limitations in group-delay measurements. Both GPS and TWSTFT rely on a group-delay measurement to transfer time and frequency information. Understanding group-delay measurements and their limitations are essential to establishing a stable method of time and frequency transfer. The simple group-delay theory in the case of a propagating sine wave is presented first and is followed by the group-delay theory of a spread spectrum signal with correlators as the detecting elements.

\section{SIMPLE GROUP-DELAY THEORY}

Let us assume a group-delay measurement in which we are trying to measure the propagation time of a signal from point $A$ to point $B$ through some non- dispersive propagation medium. Consider using a sinusoidal signal described by

$$
\sqrt{2 P} \sin (\omega t)
$$

where the power level is $P$. the frequency is $\omega$ and $t$ is time. The phase, $\phi$ of this signal after a propagation time of $\tau$, is given as

$$
\phi=\omega \tau
$$

where $\tau$ is the phase delay. The group-delay, $\tau_{g}$, is defined by

$$
\tau_{g}=\frac{\partial \phi}{\partial \omega}
$$

Since the medium is assumed to be non-dispersive, the group-delay is equal to the phase delay when there is no interfering signal. Consider the case where a signal of power $P_{m}$, with the same frequency $\omega$, delayed by time $\tau_{\alpha}$ is summed with the original signal. The phase of the interfering signal with respect to the main signal is $\theta$ described by

$$
\theta=\omega \pi_{d},
$$

where $\tau_{d}$ is the time delay between the main and interfering signal. Figure [1] shows that the combined

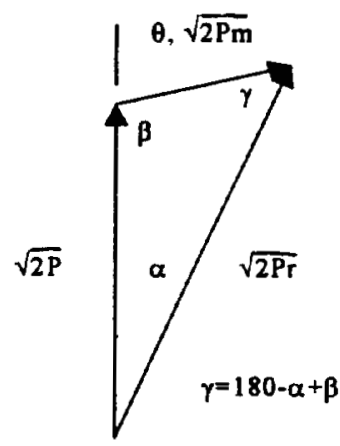

Figure 1. Vector diagram showing the relation between the main signal with power $P$, the delayed signal with power Pm, and the resultant vector sum with power Pr. 
signal has an angular deviation $\alpha$ and a new level $P_{r}$. To keep the math simple we will make the assumption that the delayed signal is small compared to the main signal. so that we can use the small angle approximation

$$
\sin (\alpha) \approx \alpha
$$

Starting with

$$
\sin (\alpha)=\sqrt{\frac{P_{m}}{P}} \sin (\gamma),
$$

we get. after using some trigonometric identities and the small-angle approximation an expression for $\alpha$ described by

$$
\alpha \approx-\sqrt{\frac{P_{m}}{P}} \sin \left(\omega \tau_{d}\right) .
$$

This expression for $\alpha$ is used to obtain the group-delay variation.

$$
\Delta \tau_{g} \approx \frac{\partial \alpha}{\partial \omega} \approx-\sqrt{\frac{P_{m}}{P}} \tau_{d} \cos \left(\omega \tau_{d}\right) .
$$

$\Delta \tau_{g}$ is the difference between the phase delay and the group-delay when there is time delayed coherent interference. For example, if the group-delay were being measured to try to determine the electrical delay of a cable, $\Delta \tau_{g}$ would be the time error due to VSWR in the cable being measured. The group-delay variation has a peak value that varies linearly with $\tau_{d}$, and goes through a full cycle when the phase between the main

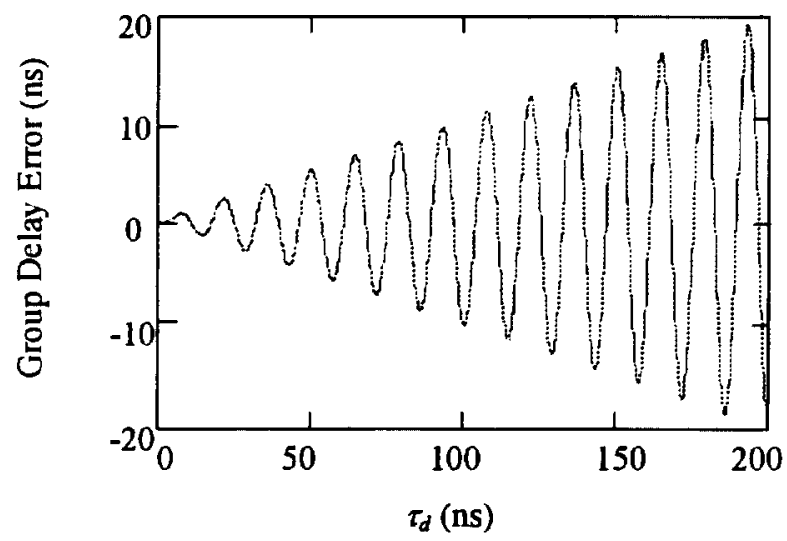

Figure 2. The group delay error $\Delta \tau_{g}$ as a function of the delay between the main and interfering signal $\tau_{d}$ for the an interfering signal level $-20 \mathrm{~dB}$ from the main signal.

signal and the delayed signal goes through $2 \pi$. The period of $\Delta \tau_{g}$ is equal to the period of the carrier frequency $\omega$. The conclusion is that a group-delay measurement may not be a good estimate of the time delay in the presence of delayed coherent interference. For large $\tau_{d}$ the peak group-delay diverges from the phase delay without bound even for small values of $P_{m}$. Figure 2 is a plot of $\Delta \tau_{\mathrm{g}}$. for increasing delay $\tau_{d}$. between the main and interfering signal.

\section{EARLY/LATE CODE CORRELATOR}

Using a spread spectrum modulated signal to measure the group-delay will limit the group-delay variations due to coherent interference. The groupdelay deviation from the phase delay goes to zero as the time delay $\tau_{d}$. between the main signal and delayed signal becomes larger and the delayed signal is no longer correlated with the main signal. An example of a system that uses a spread spectrum signal to measure time delays is GPS. For GPS. the delayed coherent interference could be due to multipath effects. Multipath signals can be large, in some cases the multipath signals may be only $10 \mathrm{~dB}$ below the direct signal. Multipath effects are well documented and the following derivations can be found in many publications [2.3]. The results can be generalized to cover delayed coherent interference from any source such as: multipath, reflections due to impedance mismatch in long antenna cables, multiple reflections through filters, near-field reflections in antennas, and reflections in waveguides. A simplified model of a noncoherent early/late (E/L) correlator such as is used in both GPS and TWSTFT systems is described below. The complete derivations of these equations are

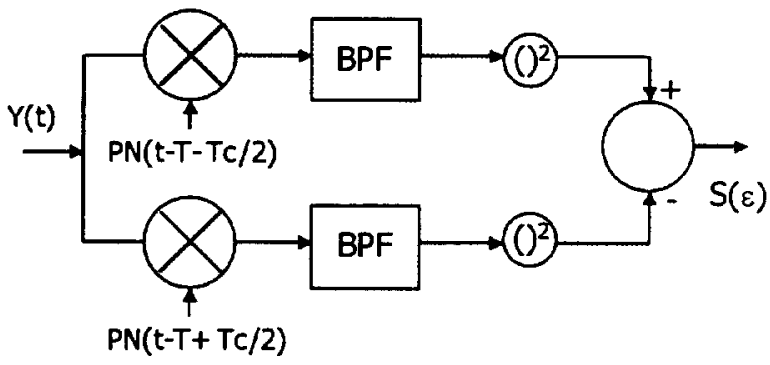

Figure 3. Code loop detector model.

presented in [2,3]. In Figure 3, the E/L code loop detector is displayed in its simplest form. The input signal $Y(t)$ is split and is the input to the early and the late correlator channels. The reference pseudo-random code sequence (PN) to the early and late correlators is spaced by a chip. The time variable is $t$, the reference sequence time estimate is $T$, and the chipping period is $T_{c}$. In both the early and late correlation channels, the output of the correlator is band-pass filtered and 
squared. The code detector error signal $S(\varepsilon)$ is the difference of the early and late correlation channel oui.. its. The error signal $S(\varepsilon)$ is driven to 0 by the delay locked loop (DLL) in normal tracking operation. The tracking-loop error (t-T) is given by $\varepsilon$.

\section{MEASURING GROUP-DELAY WITH A CORRELATOR}

First we consider a group-delay measurement without an interfering signal. The spread-spectrum signal is propagating from $A$ to $B$ in a non-dispersive medium. The spread spectrum signal is

$$
\sqrt{2 P} \cdot P N(t) \cdot \sin (\omega \cdot t)
$$

where $P$ is the power level of the signal. $P N(t)$ is the pseudo-random code sequence and $\omega$ is the carrier frequency. After a propagation time $\tau$, the signal

$$
Y(t)=\sqrt{2 P} \cdot P N(t-\tau) \cdot \sin (\omega \cdot t-\omega \tau),
$$

is received and decorrelated by the $E / L$ correlator. The error signal $S(\varepsilon)$ is given by

$$
S(\varepsilon)=P \cdot R_{P N}^{2}\left(\varepsilon-\frac{T_{c}}{2}\right)-P \cdot R_{P N}^{2}\left(\varepsilon+\frac{T_{c}}{2}\right),
$$

where $R_{P N}(t)$ is the auto-correlation function of $P N(t)$ and $\varepsilon$ is defined as the code timing error. The code timing error is

$$
\varepsilon=\tau_{g}-\tau
$$

where $\tau_{g}$ is the measured group-delay and $\tau$ is the propagation delay as defined above. Figure 4 shows the

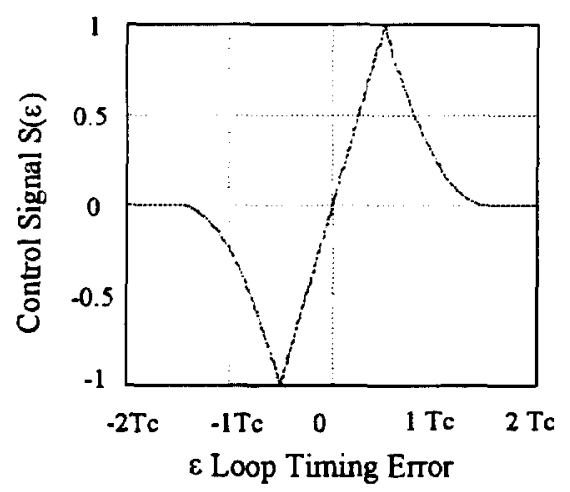

Figure 4. $S(\varepsilon)$ without interference. code detector error signal for the case of no interference. When the delay-locked loop is locked the function $S(\varepsilon)$ is equal to 0

$$
\begin{gathered}
P \cdot R_{P N}^{2}\left(\tau_{s}-\tau-\frac{T_{c}}{2}\right)=P \cdot R_{P N}^{2}\left(\tau_{g}-\tau+\frac{T_{c}}{2}\right), \\
\tau_{g}=\tau,
\end{gathered}
$$

and $\tau_{\mathrm{g}}$ measures the desired quantity $\tau$.

Now consider the case where there is a coherent interfering signal that is delayed by $\tau_{d}$. The input signal $Y(t)$ is the sum of the direct signal and one delayed interfering signal:

$$
\begin{aligned}
& Y(t)=\sqrt{2 P} \cdot P N(t-\tau) \cdot \sin (\omega \cdot t+\omega \tau) \\
& +\sqrt{2 P_{m}} \cdot P N\left(t-\tau-\tau_{d}\right) \cdot \sin (\omega \cdot t+\omega \tau+\theta),
\end{aligned}
$$

where $\tau$ is the propagation time. $\omega$ is the direct signal carrier frequency and $\tau_{d}$ is the time delay of the reflected interfering signal with respect to the direct signal. $\theta$ is the phase of the interfering signal with respect to the main signal and is related to $\tau_{d}$ by equation (4). The error signal from the code loop detector $S(\varepsilon)$ is

$$
\begin{aligned}
& S(\varepsilon)=P \cdot R_{P N}^{2}\left(\varepsilon-\frac{T_{c}}{2}\right)+P_{m} \cdot R_{P N}^{2}\left(\varepsilon+\tau_{d}-\frac{T_{c}}{2}\right) \\
& +2 P \cdot R_{P N}\left(\varepsilon-\frac{T_{c}}{2}\right) \cdot P_{m} R_{P N}\left(\varepsilon+\tau_{d}-\frac{T_{c}}{2}\right) \cdot \cos \left(\omega \tau_{d}\right) \\
& -P \cdot R_{P N}^{2}\left(\varepsilon+\frac{T_{c}}{2}\right)-P_{m} \cdot R_{P N}^{2}\left(\varepsilon+\tau_{d}+\frac{T_{c}}{2}\right) \\
& -2 P \cdot R_{P N}\left(\varepsilon+\frac{T_{c}}{2}\right) \cdot P_{m} R_{P N}\left(\varepsilon+\tau_{d}+\frac{T_{c}}{2}\right) \cdot \cos \left(\omega \tau_{d}\right) .
\end{aligned}
$$

The error signal is a function of $\tau_{d,}$, the time delay between the direct and reflected signal and $P_{m} / P$ the ratio of reflected signal power to direct signal power. Figure 5 shows the code detector error signal for the

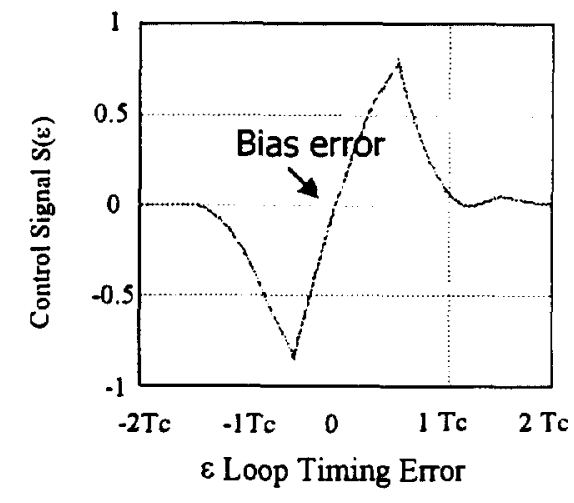

Figure 5. $S(\varepsilon)$ with interference.

U. S. Government work not protected by U. S. copyright. 
case where interference is present.

During tracking mode $S(\varepsilon)$ is 0 and $\varepsilon$ is not 0 as in the case where there is no interference. The groupdelay error $\Delta \tau_{g}$ is obtained by solving for $\varepsilon$ with the condition $\mathrm{S}(\varepsilon)=0 . \Delta \tau_{\mathrm{g}}$ is a complicated function of $P$. $P_{m} a$ and $\tau_{d}$. For $\tau_{d}<0.5 \mathrm{~T}_{c}$, the expression for $\Delta \tau_{\mathrm{g}}$ is manageable and is given by

For $P_{m}<<$. and when $\tau_{d}<0.5 T_{i}$. Equation (17)

$$
\Delta \tau_{g}=-\tau_{d}\left[\frac{P_{m}+\sqrt{P P_{m}} \cos \left(\omega \tau_{d}\right)}{P+P_{m}+2 \sqrt{P P_{m}} \cos \left(\omega \tau_{d}\right)}\right] .
$$

reduces to

$$
\Delta \tau_{g} \approx-\tau_{d}\left[\sqrt{\frac{P_{m}}{P}} \cos \left(\omega \tau_{d}\right)\right],
$$

which is identical to the expression for the sine wave signal (see Equation 9). The function for the groupdelay error changes when $\tau_{d}$ becomes larger than $T_{c}$. With a spread spectrum signal the interfering signal begins to lose correlation with the main signal as $\tau_{d}$ becomes larger than the chip spacing of the correlator (in this example $T_{c}=400 \mathrm{~ns}$ ). A plot of the group-delay error as a $\tau_{d}$ varies from zero to $1.5 T_{i}$, is shown in Figure 6 for the case of an interfering signal that is -20 $\mathrm{dB}$ lower in power than the direct signal. The carrier

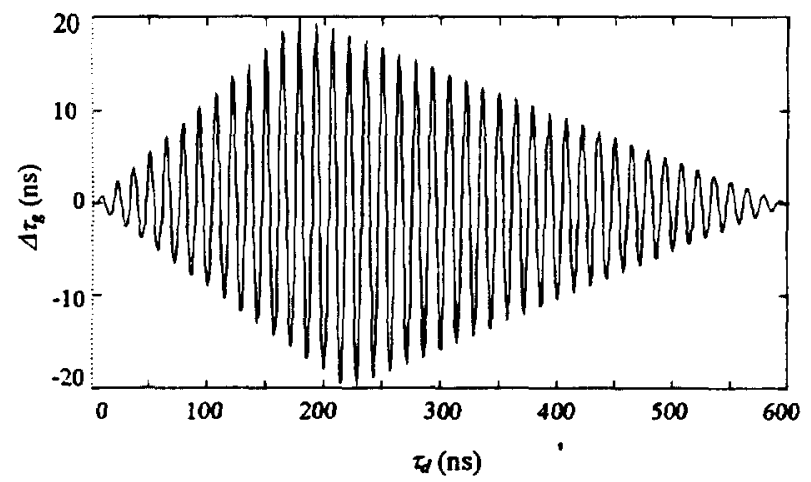

Figure 6. Group delay error as a function of $\tau d$. The chip period is $400 \mathrm{~ns}$ and the detector is a 1/2chip E/L correlator.

frequency is $70 \mathrm{MHz}$ and the chip rate is $2.5 \mathrm{MHz}$. The error becomes smaller for $\tau_{d}>200 \mathrm{~ns}$. Understanding the group-delay error function can help us improve the spread spectrum time and frequency transfer systems. The following section discusses some possibilities.

\section{MINIMIZING GROUP-DELAY ERRORS IN SPREAD SPECTRUM SYSTEMS}

The most obvious method of reducing the group-delay error is by reducing the reflected signal power. $P_{m}$. Antennas can be positioned to reduce the effects of multipath. All impedances should be matched to minimize signal reflections in cables and system components such as filters and amplifiers. Phase stable cables should be used to minimize mapping small phase changes into large delay errors [4].

The other method is to exploit the correlation properties of the spread spectrum system. Recall that as $\tau_{d}$ becomes larger than half the clip spacing of the correlator the group-delay error begins to decrease. One method of decreasing the group-delay error is therefore to decrease the correlator spacing [3]. Decreasing the correlator spacing comes at an expense. The linear tracking region and the acquisition performance are degraded [3]. It may not be possible to operate under these conditions in cases where the signal-to-noise ratio is poor. Figure 7 shows the group-

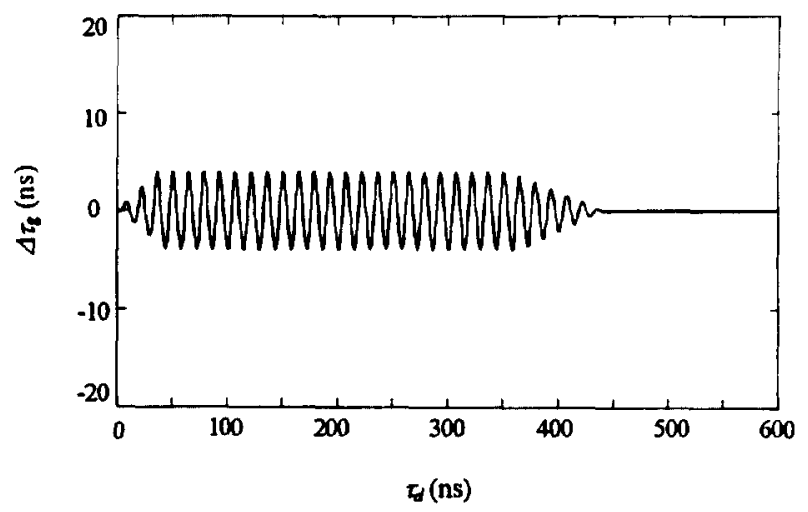

Figure 7. Group delay error as a function of $\tau \mathrm{d}$. The chip period is $400 \mathrm{~ns}$ and the detector is a $1 / 10$ chip $E / L$ correlator.

delay error as a function of $\tau_{d}$ for the same signal conditions as in Figure 6 but with a one-tenth chip $E / L$ correlator. In other words the reference code to the early and late correlators is spaced by one-tenth of a chip.

Another method of reducing the group-delay error is to use a higher chip rate. By increasing the chip rate you decrease the correlation time window during which the interfering signal affects the group-delay measurement. Figure 8 displays the group-delay error for the same signal levels as in Figure 6 but the spread spectrum signal is generated by a $20 \mathrm{MHz}$ chip rate. The E/L correlator spacing is one-half of a chip. 
In the TWSTFT system one of the sources for the group-delay error is the VSWR in the $30 \mathrm{~m}$ to $60 \mathrm{~m}$

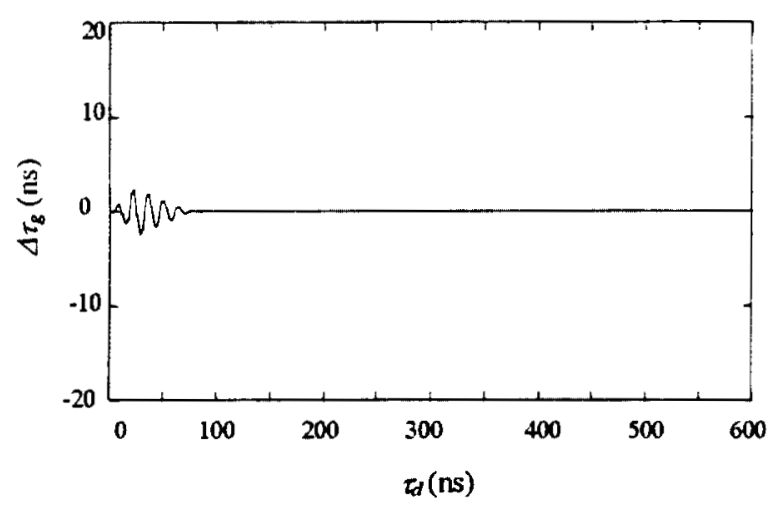

Figure 7. Group delay error as a function of $\tau d$. The chip period is 50 ns and the detector is a onehalf chip $\mathrm{E} / \mathrm{L}$ correlator.

cables that go from the laboratory to the earth station [4]. The cables lengths are such that reflected signals due to VSWR are delayed from the main signal by 300 to $400 \mathrm{~ns}$. As has been illustrated in Figure 6, the group-delay error has the potential of being large. Making the reflected signal delay larger than $600 \mathrm{~ns}$ (greater than $1.5 T_{c}$ ) could make the group-delay error smaller without changing the chip rate of the modems. This could be accomplished by using an optic fiber RF link with a propagation delay greater than $600 \mathrm{~ns}$. The delayed coherent interference caused by reflections at the ends of the fiber would be delayed by greater than 600 ns and would no longer be correlated with the direct signal. To implement this method of mitigating the group-delay error, optical fiber should be used and not a longer RF cable. Reflections in the RF cable can occur at random places along the cable due to dielectric imperfections, kinks, or temperature gradients. Reflections in optical fibers are, for the most part, constrained to occur only at the ends.

\section{CONCLUSIONS}

\begin{abstract}
All group-delay measurements are sensitive to delayed coherent interference. Group-delay measurements using a swept sine wave have a groupdelay error function that is proportional to the time delay of the interfering signal. The spread spectrum group-delay measurement systems have the advantage that the group-delay error function is 0 outside the correlator bandwidth. In addition, using the time correlation properties of spread spectrum systems can minimize the group-delay error function. Systems that suffer delay errors due to VSWR problems cannot be calibrated due to the sensitivity of the group-delay error
\end{abstract}

U. S. Government work not protected by U. S. copyright. function on the phase of the carrier. The VSWR in ground station components is closely coupled to the environment and may be the major cause of instability in TWSTFT. New technology that is now available may be used to increase the stability of current methods of time and frequency transfer. Some of this technology includes optical fiber RF links. modems that are capable of chip rates in excess of $20 \mathrm{MHz}$. phase stable coaxial cables. and better antenna designs.

\section{REFERENCES}

1. Larson, K. "Comparison of GPS Carrier Phase and Two-way Satellite Time and Frequency Transfer." to be published in Proc. $30^{\text {th }}$ Precision Time and Time Interval Systems and Applications Meetings. 1998.

2. Holmes. J. K., Coherent Spread Spectrum Systems, John Wiley and Sons. Inc.. New York. 1982.

3. Van Dierendonck. A. J., "GPS Receivers." Global Positioning Systems: Theory and Applications. Vol. 1, American Institute of Aeronautics and Astronautics, Inc., Washington, 1996.

4. Ascarrunz. F. G., Parker, T. E. and Jefferts. S. R. "Pseudo-random code correlator timing errors due to multiple reflections in transmission lines," to be published in Proc. $30^{\text {th }}$ Precision Time and Time Interval Systems and Applications Meetings, 1998. 